\title{
Intérêts régionaux et leviers nationaux : regards sur le conflit syrien par ses marges kurdes
}

\section{Félix Poyer}

\section{(2) OpenEdition}

\section{Journals}

\section{Édition électronique}

URL : http://journals.openedition.org/echogeo/13630

DOI : 10.4000/echogeo. 13630

ISSN : 1963-1197

\section{Éditeur}

Pôle de recherche pour l'organisation et la diffusion de l'information géographique (CNRS UMR 8586)

\section{Référence électronique}

Félix Poyer, «Intérêts régionaux et leviers nationaux : regards sur le conflit syrien par ses marges kurdes », EchoGéo [En ligne], Sur le Vif, mis en ligne le 15 novembre 2013, consulté le 20 avril 2019. URL : http://journals.openedition.org/echogeo/13630; DOI : 10.4000/echogeo.13630

Ce document a été généré automatiquement le 20 avril 2019.

\section{(c) (i) (9)}

EchoGéo est mis à disposition selon les termes de la licence Creative Commons Attribution - Pas d'Utilisation Commerciale - Pas de Modification 4.0 International 


\title{
Intérêts régionaux et leviers nationaux: regards sur le conflit syrien par ses marges kurdes
}

\author{
Félix Poyer
}

\section{Introduction}

Depuis la mi-juillet 2012, on observe, dans les zones kurdes de Syrie, une prise en main du territoire par les mouvements kurdes.

2 Cette prise de contrôle, réalisée à la faveur de la révolution syrienne, se manifeste par un réinvestissement de l'espace public. La réorganisation administrative opérée par le PYD (Union des partis démocratiques), pendant politique syrien du PKK (Parti des travailleurs kurdes) fondé en 1978 par Abdullah Öcalan en Turquie, contribue à la mise en place de zones qui sont aujourd'hui de facto autonomes au nord de la Syrie. Ces espaces s'étendent autour des villes d'Afrin et de Qobane (anciennement Ayn Al Arab), au contact de la frontière turque, ainsi qu'au nord-est dans la province d'Al Hassake (aussi connue sous le nom de Jezireh), au contact des frontières turques et irakiennes (cf. cartes proposées par Le Monde et Jeune Afrique). Si cette dernière zone est la plus peuplée, il ne faut pas oublier qu'une importante diaspora a constitué des quartiers majoritairement kurdes à Damas ou à Alep. Certains de ces quartiers ont été abandonnés par leurs habitants à Damas, d'autres, notamment à Alep, sont eux sous le contrôle des forces kurdes des YPG ( Yekîneyên Parastina Gel, Unités de Défense du Peuple).

3 La polarisation croissante de la Jezireh, désormais «libérée » de l'emprise damascène, vers le Kurdistan irakien, illustre la profondeur des changements en cours dans ce territoire des marges de la Syrie. Le Rojava Kurdistan (Kurdistan occidental, situé dans l'extrémité nord-est de la Syrie) saisit ainsi les opportunités qui se présentent.

4 La question qui nous intéresse ici est de déterminer de quelles dynamiques sont porteurs les Kurdes de Syrie, tant dans les évolutions de la crise syrienne que dans la perspective 
de l'établissement d'un hypothétique Kurdistan. Un décryptage des différents acteurs et de leur position respective permettra de mettre en lumière les options qui aujourd'hui se posent pour un « Kurdistan syrien ».

5 Ce travail s'appuie sur un séjour de terrain en mai 2013 dans la région de la Jezireh en compagnie du journaliste Pierre Torrès, actuellement retenu en otage en Syrie, dont nous présentons ici quelques photographies. Cet article présente des observations et des informations récoltées dans un contexte encore très instable. Il n'a pas prétention à l'exhaustivité mais consiste davantage en quelques pistes de réflexions pour appréhender la situation actuelle et les évolutions envisageables à court ou à moyen terme.

\section{Origines et mutation de la question kurde en Syrie et dans les pays limitrophes}

6 Forts d'une population estimée entre 25 et 37 millions d'individus, les Kurdes sont, aujourd'hui encore, le "peuple sans État» le plus nombreux. Cette situation, relativement ancienne, a pour origine les accords de Lausanne conclus en 1923 entre les puissances européennes et les Turcs lors du démantèlement de l'Empire Ottoman (Boulanger, 2006).

7 Cette question du "peuple sans État» est une problématique transfrontalière et, par conséquent, régionale. Les Kurdes sont, en 2006, 13,1 millions en Turquie (21\% de la population totale du pays), 9,2 millions en Iran (14\%), 4,7 millions en Irak (18\%), 1,2 million en Syrie $(8 \%)^{1}$. Si l'on ajoute à cela le poids de la diaspora estimée à 3 millions de personnes dans le monde, dont 1 million en Europe, on peut considérer que cette question identitaire relève désormais, en termes sociaux et culturels, des conséquences de la mondialisation (Boulanger, 2006)

8 Les enjeux stratégiques que sont désormais l'eau et le pétrole (illustration 1) dans la géographie du Proche-Orient ne manqueront pas de conférer à la Jezireh, région marginale de la Syrie, une position clé dans la péninsule. 


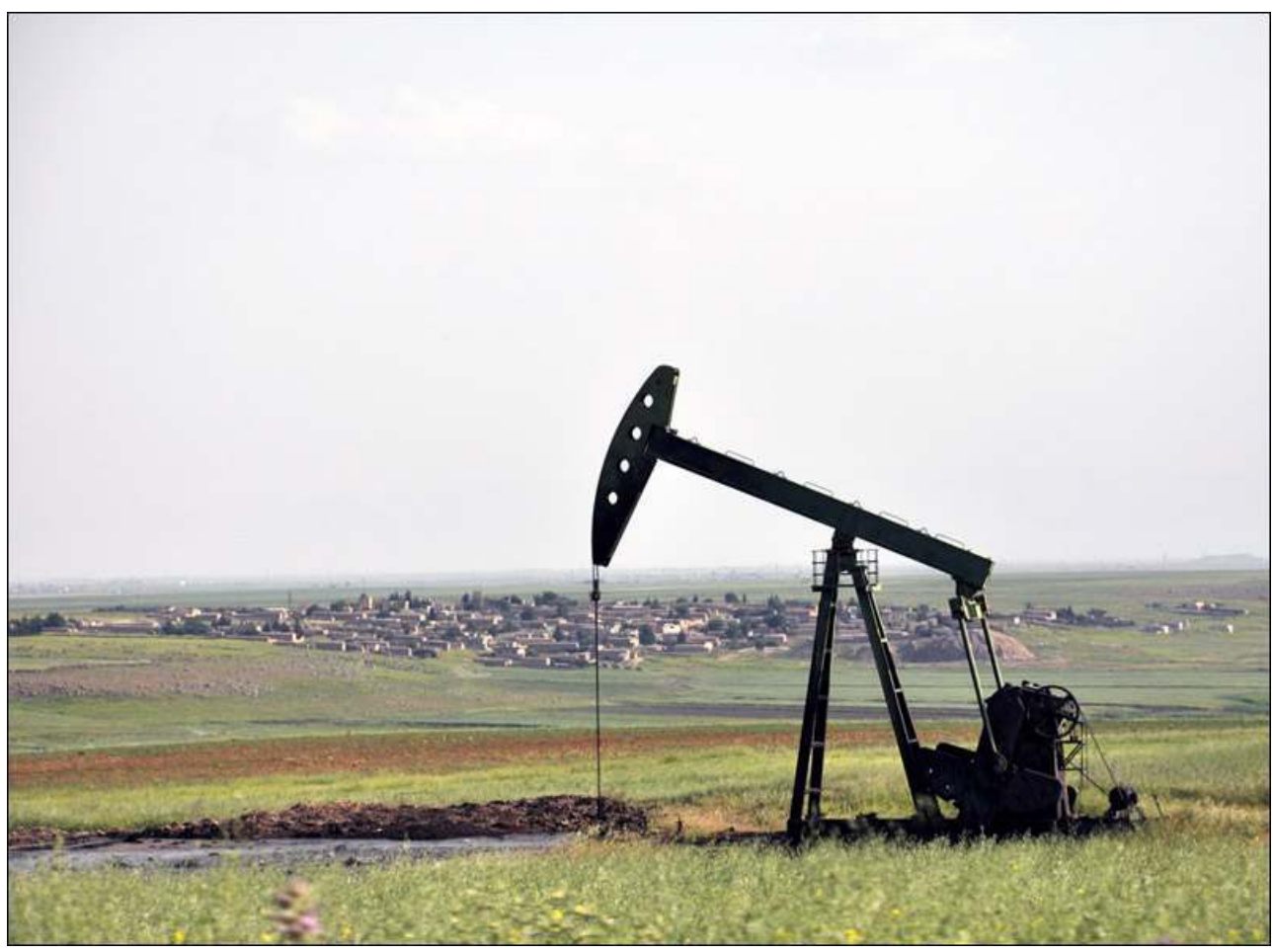

Auteur : P. Torrès, mai 2013.

La richesse en pétrole de la Jezireh est sujette à controverse. Qui connait l'étendue de sa production? S'il n'est possible d'accéder à aucune donnée de cette nature, le paysage n'en est pas moins fortement marqué par les infrastructures liées à l'extraction et au transit de cette énergie fossile. Le poids prépondérant du pétrole dans l'économie des voisins kurdes d'Irak, grâce à la présence de multinationales américaines et aux besoins $\mathrm{du}$ voisin turc, laisse supposer que la question énergétique peut permettre aux populations de disposer de soutiens déterminants tant économiques que diplomatiques dans la construction de leur autonomie, pour peu que ces ressources soient significatives.

La légitimité du KRG (Kurdish Regional Government ou Kurdistan d'Irak, aujourd'hui largement autonome et pacifié) à participer à l'avenir du Rojava réside avant tout sur son poids diplomatique croissant dans la région. Sa proximité avec la Turquie en fait un médiateur-clé pour assurer le devenir d'un éventuel Kurdistan syrien (Park, 2012). De plus, l'afflux massif de réfugiés kurdes sur le sol du KRG, en provenance de toute la Syrie,

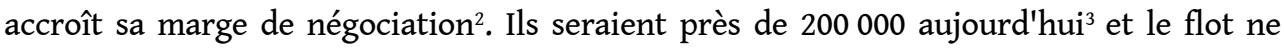
semble pas se tarir. Renforcé par la recrudescence d'affrontements depuis juin 2013 avec les djihadistes d'État Islamique d'Irak et du Levant, les mouvements de réfugiés traduisent les nouvelles polarités qui s'offrent aux kurdes syriens.

« À présent, les familles [kurdes de Syrie] qui arrivent doivent parfois attendre plus d'une semaine avant d'obtenir tentes et matelas. Le camp sature et la logistique ne suit pas. [...] Nous avons besoin d'un plus large soutien au niveau international » (entretien avec le responsable des relations extérieures du camp de Domiz dans le gouvernorat de Dohouk au Kurdistan d'Irak, le 08 mai 2013).

11 Le camp de réfugiés de Domiz (illustrations 2 et 3), dans le gouvernorat de Dohouk au Kurdistan d'Irak, a été ouvert par le gouvernement régional kurde en avril 2012 pour 
accueillir les réfugiés kurdes de Syrie. Situé à $60 \mathrm{~km}$ de la frontière syrienne, ce camp abrite actuellement 90000 des 160000 syriens réfugiés en Irak, un chiffre dépassant largement les 30000 personnes initialement attendues. Face à cette situation imprévue, les autorités kurdes d'Irak développent des infrastructures de plus grande ampleur, telle cette route en construction qui reliera le camp à Dohouk, tendant à la pérennisation de ce camp. Trois autres camps ont été ouverts au Kurdistan d'Irak, deux à Erbil, capitale et fief du PDK de Massoud Barzani, un dernier à Souleimaniye, fief de l'UPK de Jalal Talabani, l'autre parti de gouvernement au Kurdistan d'Irak.

Illustration 2 - Le camp de réfugiés de Domiz, vue sur le chantier

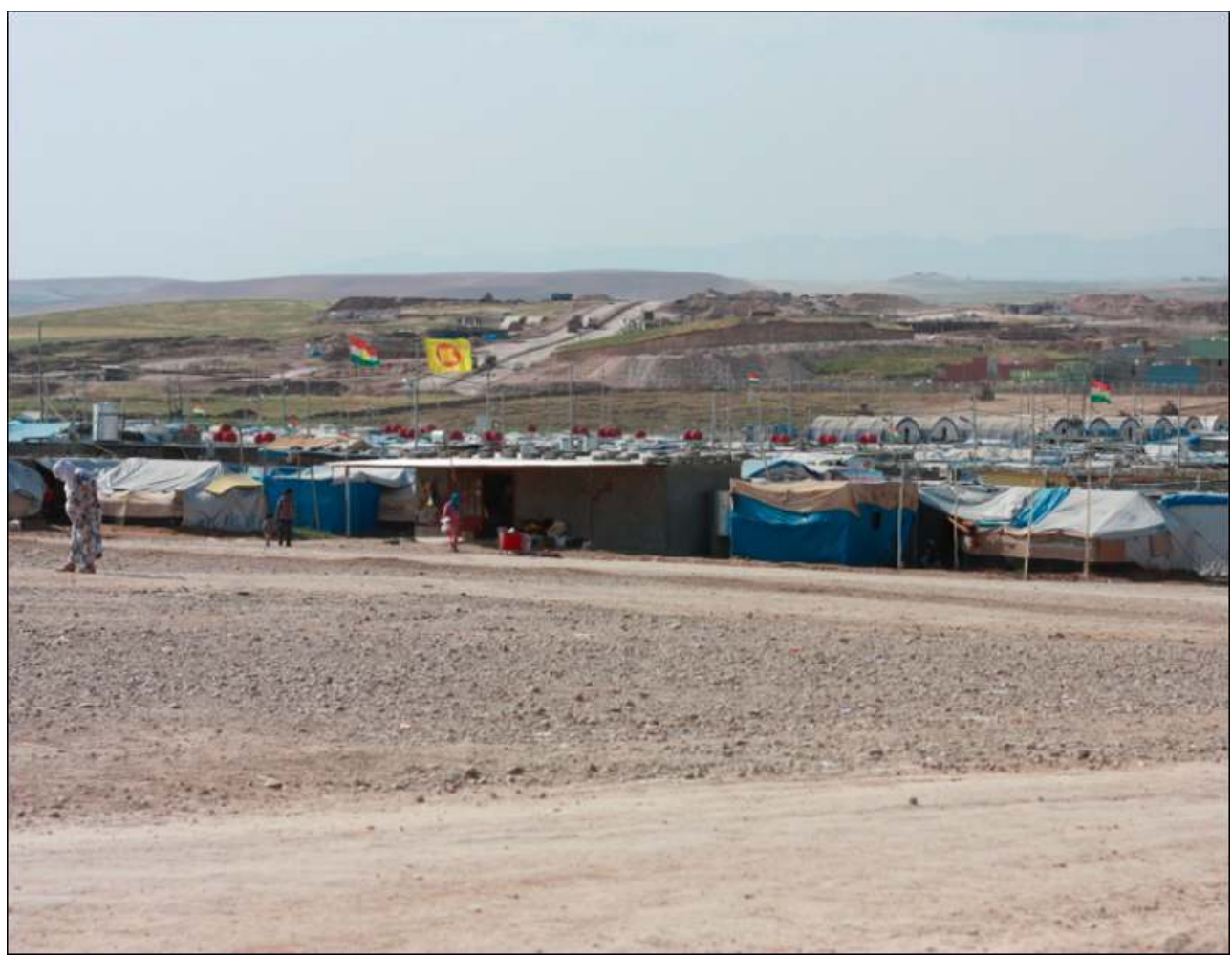

Auteur : F. Poyer, mai 2013.

Face à la croissance du camp, des infrastructures plus conséquentes que celles initialement prévues sont mises en chantier. Enjeu crucial de ces mutations, l'approvisionnement devient synonyme de pérennisation... 


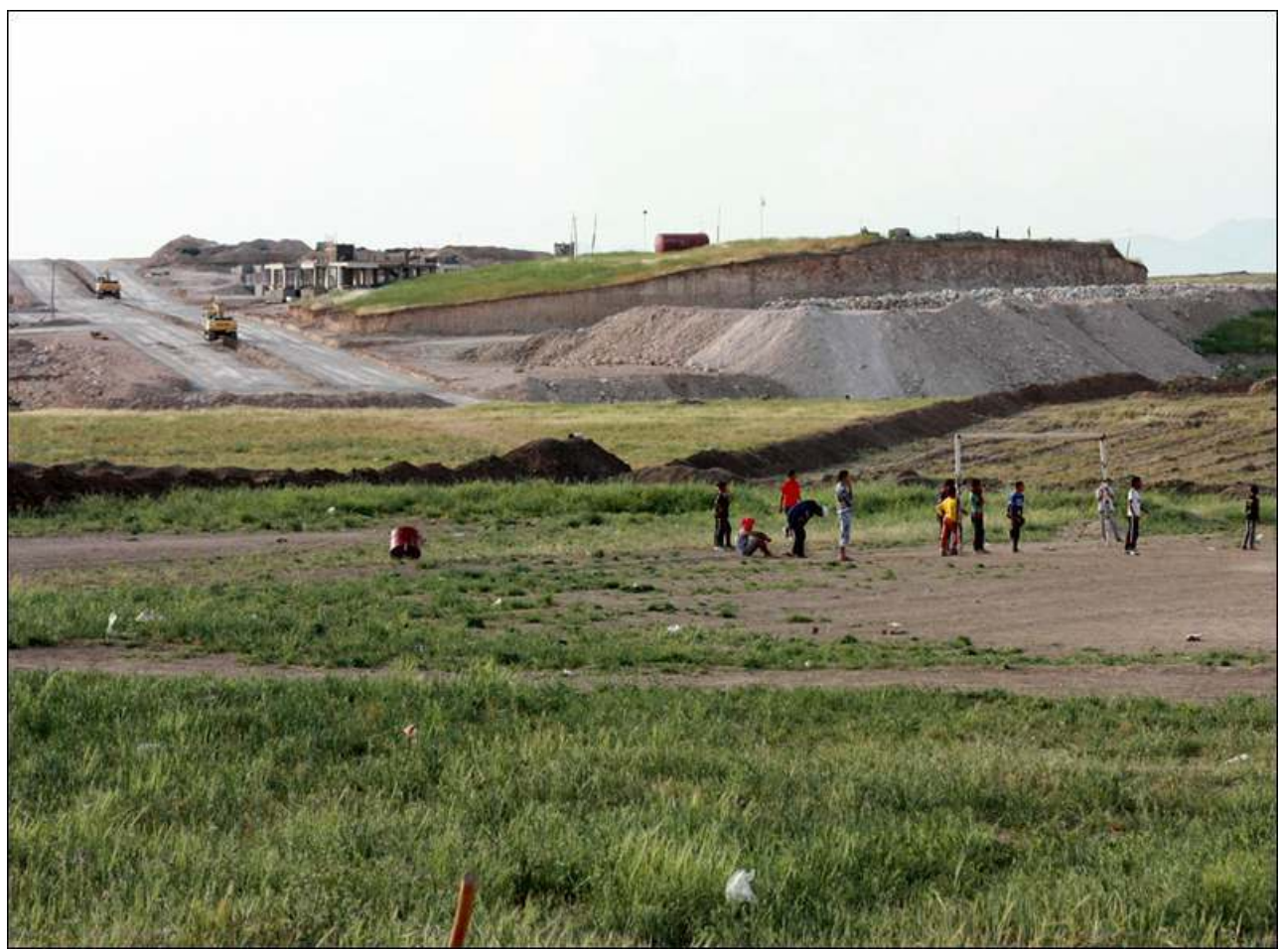

Auteur : F. Poyer, mai 2013.

Si la question kurde traverse le $\mathrm{XX}^{\mathrm{e}}$ siècle, elle se pose, depuis une vingtaine d'années, en des termes nouveaux dans la Région (Barzani, 2008). Ainsi, le Kurdistan d'Irak jouit à présent d'une large autonomie, officiellement établie par la constitution irakienne de 2005, sous la houlette du Parti Démocratique du Kurdistan (PDK) et de l'UPK. Parallèlement, les Kurdes de Turquie bénéficient d'un timide élargissement de leurs droits culturels mis en œuvre par l'AKP (Parti pour la justice et le développement, au pouvoir en Turquie depuis 2002), premier parti de gouvernement à reconnaître l'existence d'un « problème kurde » en Turquie ${ }^{4}$.

En Syrie, la question nationale kurde est formalisée en 1957, avec la création du PDKS (Parti démocratique kurde de Syrie), un parti d'opposition né de l'expérience irakienne et de l'impulsion directe du PDK. Le régime baathiste syrien, dès sa prise du pouvoir en 1963, adopte des mesures discriminatoires à l'encontre des Kurdes. L'arrivée au pouvoir d'Hafez Al-Assad en 1970 marque une rupture dans la gestion du "problème kurde ", en tournant le nationalisme kurde à son avantage, par un jeu d'alliances stratégiques réalisées avec le PDK en Irak puis le PKK en Turquie (Gauthier, 2006). Outils de sa politique de déstabilisation régionale, les Kurdes de Syrie mettent en pratique leurs velléités nationales par la lutte armée dans les pays voisins, conformément aux intérêts momentanés du régime. En 1998, face aux pressions répétées des Turcs, Damas lâche le PKK et son leader, Abdullah Öcalan, est contraint de fuir le pays.

Cette politique de détournement de la question kurde s'appuyait en Syrie sur une ambivalence entre l'intégration d'une fraction des élites kurdes au plus haut niveau de l'État (Gauthier, 2006) et l'oppression d'un peuple privé de droits culturels et même, pour 150000 de ses membres, de nationalité depuis 1963 (Bozarslan, 1997, p. 187-190). En dépit de ce système, la question kurde a su opérer un retour sur la scène nationale syrienne, 
dans la foulée du fugace «printemps de Damas » des années 2000 (Burgat, 2013), durant lequel le nouveau président Bachar Al-Assad avait temporairement soulevé la chape de plomb baathiste pesant sur le pays.

16 Les manifestations en faveur des droits culturels des Kurdes, si restreintes fussent-elles en 2002 et 2003, puis les émeutes de Qamishlo en 2004, ont marqué le passage de la question nationale kurde en Syrie de la « dissimulation à la « visibilité », selon la formule avancée par Jordi Tejel Gorgas (2006).

«Les Kurdes syriens [le 11 mars 2004] ont manifesté d'eux-mêmes. L'erreur des partis est d'être à l'époque restés divisés. Le Régime a été plus fort que le mouvement. Nous ne devons pas reproduire ce scénario »

(entretien de l'auteur avec un journaliste syrien de Zagros TV, chaîne kurde basée à Erbil au Kurdistan d'Irak et proche du PDK, le 5 mai 2013).

17 Ces mouvements protestataires, dont celui initié en 2011 dans l'ensemble de la Syrie, ont mis au jour une tension entre d'une part la jeunesse kurde tendant à la radicalisation de ses revendications et d'autre part les partis politiques, rendus suspects par leur tendance au compromis avec le régime.

Illustration 4 - Graffiti en kurde sur la route de Qamishlo (anciennement Qamishli)

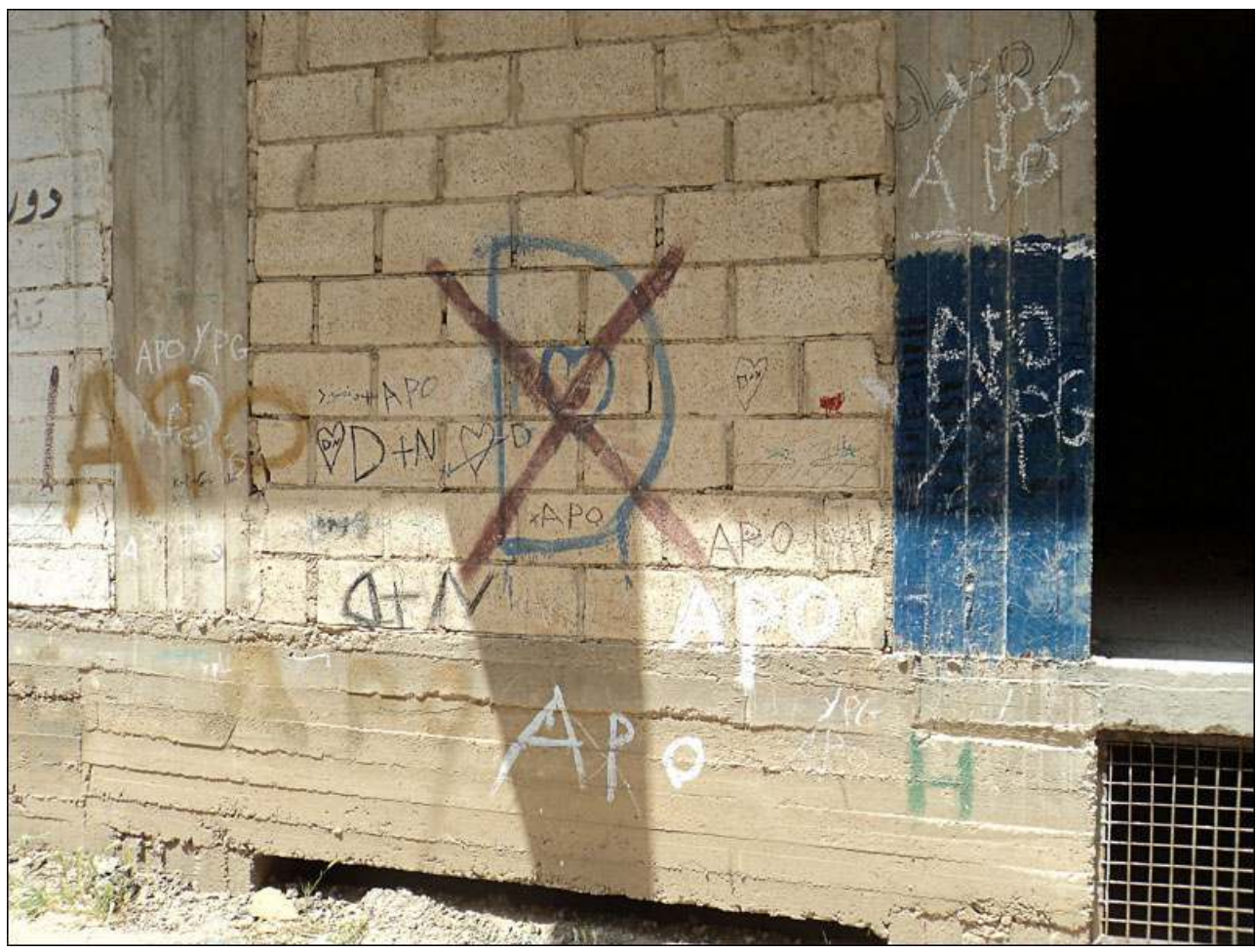

Ici : inscriptions à la gloire d'Abdullah Öcalan, le fondateur du PKK, surnommé « Apo »

Auteur : F. Poyer, mai 2013.

18 L'affirmation de la visibilité de l'identité kurde passe par le rejet des toponymes arabisés depuis les années 1960. La multiplication de graffitis en langue kurde (illustration 4) et la foison de drapeaux aux couleurs du Kurdistan témoignent d'une indéniable affirmation nationaliste dans les trois zones de peuplement kurdes en Syrie que sont la Jezireh (Gouvernorat d'Hassake), Qobane et Idlib. Dans la ville de Girkê Legê, les graffitis inscrits dans les premiers jours du déploiement des forces kurdes témoignent de l'influence, en leur sein, du PKK. Partout fleurirent des inscriptions à la gloire d'Abdullah Öcalan, le 
fondateur du PKK. "Apo ", qui signifie oncle en Kurmandji, est en effet le surnom du leader charismatique de cette guérilla trentenaire. Si à présent les forces kurdes (YPG) tentent de masquer leur appartenance au PKK lorsqu'elles communiquent avec des représentants des médias internationaux, l'euphorie des premiers temps a laissé sa marque sur le paysage urbain de la Jezireh.

Cependant, les mutations de la contestation populaire de 2011 en résistance armée puis en guerre civile ont considérablement renforcé la position des partis politiques kurdes auprès de leur population. Les moyens qu'ils ont mis en œuvre pour organiser le changement et capitaliser le soutien populaire leur confèrent aujourd'hui une position ambiguë sur l'échiquier syrien, résultat du détournement de la contestation populaire anti Bachar al-Assad vers un mouvement autonomiste.

\section{De la révolution syrienne à une révolution kurde}

La Syrie, pays pluriethnique et multiconfessionnel, a bâti une identité unitaire fondée sur la nation. Cette construction, dont le parti Ba'ath se voulait la pierre angulaire, n'était pas démentie par les manifestants de 2011 qui, grisés par les révolutions arabes, tentèrent d'imposer la chute du régime et de son système répressif. Les slogans utilisés lors de ces manifestations indiquaient clairement l'unité des participants, en affirmant la primauté d'une identité syrienne transcendant toute autre construction identitaire, infra ou extra étatique. Cependant, après deux années de guerre, la lecture du conflit prend globalement une apparence confessionnelle (principalement entre chiites et sunnites) et, localement, une apparence ethnique, confortant la position des nationalistes kurdes.

Cette évolution de la révolution syrienne, opérée à la faveur du passage à la lutte armée, n'est pas sans fondement stratégique. Ainsi l'opportunité d'une révolution pankurde au sein de la révolution syrienne est-elle révélatrice, à l'échelle infra-nationale, des réponses apportées par le gouvernement de Bachar Al-Assad.

Comme ailleurs en Syrie, les protestations en faveur de la chute du régime se sont déclarées massives et populaires dès leurs prémices, en mars 2011 (Yazbek, 2012). Mais l'attitude des Kurdes reste réservée face à ce mouvement, puisqu'il est soutenu par la Turquie.

23 De la même manière, le régime a, à plusieurs reprises, fait preuve de plus de discernement dans la répression des manifestations dans les zones de peuplement kurde que dans les zones arabes ${ }^{5}$. La répression cible les meneurs plutôt que les foules, la pression par les bombardements aériens reste étonnamment mesurée, même si elle est maintenue encore à l'heure actuelle, rappelant que le régime garde la suprématie sur l'espace aérien.

"Quand ils [les forces loyalistes] bombardent par voie aérienne le village de Tall Hadad, ce n'est pas pour atteindre un objectif sur le terrain. C'est pour rappeler aux Kurdes que les avions sont là, et qu'ils peuvent frapper n'importe où, n'importe quand. [...] Les YPG ne disposent pas d'armement anti-aérien, ils ne peuvent protéger le Rojava de cette menace »

(entretien avec un journaliste citoyen à Qamishlo, le 7 mai 2013).

En dépit du caractère unitaire de l'opposition, un seul parti kurde participe à la fondation du Conseil national syrien (regroupement des partis d'opposition organisé à Istanbul avec l'aide du gouvernement turc) : le Mouvement du Futur, fondé par Mishaal Tamo en 2005. Accusé à ce titre d'être une voix discordante au sein des partis politiques kurdes de Syrie 
et de pactiser avec la Turquie, Tamo sera assassiné peu après son rapprochement avec le $\mathrm{CNS}^{6}$.

Pour l'État syrien, la mise en place d'un foyer kurde de déstabilisation le long de la frontière turque est une réponse à ce qu'il dénonce comme des manœuvres de la Turquie dans l'agitation syrienne ${ }^{7}$. Lorsqu'en juillet 2012 les forces gouvernementales opèrent un retrait des principales villes de Jezireh, cédant la place aux milices kurdes du PYD, la situation se trouble 8 . Le PYD, antenne du PKK, tirerait-t-il à l'origine sa position dominante sur l'échiquier kurde syrien d'accords passés avec Damas ? Ce qui est sûr, c'est que la Syrie baathiste ne peut qu'apprécier le trouble induit par la création d'une base arrière pour le PKK, au grand dam de la Turquie'.

Quoiqu'il en soit, l'analyse de Rhodi Mellek, représentante et porte-parole du PYD à Bruxelles, évoquant un redéploiement des forces loyalistes syriennes «là où elles sont plus utiles", est emblématique de la gestion de la crise mise en œuvre par le gouvernement syrien. La stratégie de contre-insurrection du régime a su, d'une part, favoriser la division des forces ennemies. D'autre part, le repli des forces gouvernementales syriennes sur l'axe stratégique Alep-Damas constitue un moyen d'accentuer la pression de ses propres forces sur la «surface utile» du pays, selon une stratégie de guerre contre-révolutionnaire progressive et compartimentée qui n'est pas sans rappeler les travaux désormais célèbres de David Galula, réalisés en 1963 et traduits en français en 2008, à la faveur de la guerre d'Irak (Galula, 2008).

C'est dans ce contexte particulier que la prise en main du territoire par les milices kurdes doit être perçue. Si en temps de paix le rapport de force entre le gouvernement syrien et les forces kurdes ne pouvait permettre de tels déploiements, la nécessité pour le régime de ne pas ouvrir un nouveau front a rendu possible le déploiement des forces du PKK dans les zones kurdes de Syrie.

Les équilibres restant précaires, le PYD, désireux de renforcer son implantation dans la zone, se voit obligé de maintenir la contestation à un niveau tolérable pour Damas, afin de s'épargner une situation de guerre ouverte avec le régime. La suprématie aérienne de Damas reste une épée de Damoclès non négligeable pour la survie du mouvement kurde. Les accords d'Erbil conclus en 2012 entre l'ensemble des partis kurdes le stipulent: les accords affirment rejeter la violence ainsi que «toute pratique conduisant à l'accroissement des tensions dans les zones kurdes » (art. 5 des accords d'Erbil). Est-ce en vertu de ces principes que le PYD a réprimé diverses manifestations pacifiques prônant la chute du système baathiste, comme à Amouda, où il a fait tirer sur la foule ${ }^{10}$ ?

En plus de cet apport stratégique pour le régime, en termes de pression géopolitique et de désinvestissement d'une région périphérique, le gouvernement syrien a su profiter des revendications portées par les Kurdes. Pour apparaitre aux yeux de l'opinion internationale comme un gouvernement favorable aux minorités et bénéficiant de leur soutien, Damas avait annoncé, dès avril 2011, la naturalisation de quelques 300000 kurdes, jusqu'ici apatrides ${ }^{11}$. Cette annonce, de même que celle de l'ouverture d'une chaire de littérature kurde à l'université de Damas en mai $2013^{12}$, associée à une intense communication dans les médias nationaux, avait fait germer l'idée, dans l'opinion nationale, d'une accointance entre le régime et les Kurdes. Certains détails, comme la destruction par les forces du PYD de documents saisis dans les locaux des services secrets du régime, ne sont pas sans légitimer ces interrogations ${ }^{13}$. Si l'on en croit plusieurs 
familles de propriétaires terriens rencontrées durant ce séjour, les récoltes de blé du gouvernorat d'Hassake, comme en temps de paix, sont achetées par Damas.

Mais les équilibres restent instables. La visite de M. Saleh Muslim, leader du PYD, à Ankara le 26 juillet 2013, laisse entendre que les Turcs sont conscients des déstabilisations induites par les groupes islamistes liés à Al Qaida dans la zone. Ces groupes, brouillant l'action de l'armée syrienne libre, ont été renforcés dans leur autonomisation par l'autoproclamation de l'État islamique d'Irak et de Cham dans l'ouest irakien, tenu par des mouvements djihadistes proches d'Al Qaida. Ainsi, le gouvernement turc pourrait bien faire évoluer sa position vis-à-vis des nationalistes kurdes. Le processus de paix en cours en Turquie entre le gouvernement et le PKK, si chaotique soit-il ${ }^{14}$, semble devoir déjà beaucoup aux événements récents. Les troubles récents ont offert à l'État turc de transformer sa gestion interne du problème PKK.

Nouveau facteur d'incertitude, la rupture, fin juin 2013, entre l'Armée Syrienne Libre et certains de ses éléments djihadistes ${ }^{15}$ affaiblit l'opposition armée au régime. Ce bouleversement des équilibres pourrait conduire la Coalition Nationale Syrienne, en dépit d'affrontements internes passés et de suspicions réciproques ${ }^{16}$, à plus de souplesse vis à vis des exigences kurdes, quant aux projections d'une Syrie post-Assad.

«Au début [2011], nous voulions travailler avec l'opposition syrienne, mais celle-ci, aujourd'hui encore, ne nous offre pas suffisamment de garanties. En dépit de cela, notre vision pour la Syrie reste d'ampleur nationale "

(entretien de l'auteur avec un membre du bureau du PDPKS (Pes Vero), parti kurde fondé en 1957 et signataire des Accords d'Erbil, Girkê Legê, le 3 mai 2013).

De récents rapprochements semblent aller dans ce sens ${ }^{17}$, les forces kurdes affrontant les djihadistes depuis déjà plusieurs mois ${ }^{18}$. Mais la grande volatilité des équilibres dans la guerre civile ne permet pas, en l'état actuel des choses, de présager des suites à ces premiers pas. Le simple fait que des contacts soient établis constitue déjà une avancée remarquable.

«Mon père nous a dit, au début de l'insurrection, que l'armée libre ne menait pas notre combat. Le nôtre, nous disait-il, est de combattre pour le Kurdistan. Je réponds : "Contre qui" ? Il me dit qu'il ne sait pas »

(entretien avec un jeune pacifiste, membre d'une organisation de Kurdes et d'Assyriens, active depuis 2011, le 4 mai 2013).

\section{Vers quel Kurdistan?}

Comme nous l'avons vu précédemment, le parti de facto aux commandes dans les zones de peuplement kurdes de Syrie est étroitement lié au PKK. L'expérience militaire et les capacités organisationnelles du PKK ont permis un déploiement rapide de forces structurées, sanctuarisant le territoire. Cependant, le PKK étant considéré par certains pays comme une organisation terroriste, on comprend que le PYD ne peut disposer d'autres relais à l'international que de son propre réseau de soutiens, composé entre autres d'ONG et de sites web d'information. Si ce réseau de soutien lui assure une remarquable maitrise de l'information, atout remarquable dans le cadre d'une guerre insurrectionnelle, sa position déficitaire sur le plan diplomatique constitue pour le mouvement un handicap majeur. C'est pour ce parti un premier enjeu stratégique.

En second lieu, si le PYD dispose d'une suprématie dans les zones de peuplement kurde, la population ne lui est acquise que de manière parcellaire. Les taxes levées sur les réfugiés et sur le transit des marchandises contribuent à lui conférer une certaine impopularité. 
C'est dans ce contexte qu'ont été conclus les accords d'Erbil, en juillet 2012, sous le patronat de Massoud Barzani. Ces accords concrétisent la volonté de réunir l'ensemble des partis kurdes au sein d'une coalition unitaire, le Conseil Suprême Kurde. Pourtant, la position dominante du PYD sur le terrain lui permet de se tailler la part du lion. Si le Conseil National Kurde obtient 5 sièges dans cette instance, le PYD et ses affidés en obtiennent le même nombre, ce qui lui assure une représentation très favorable ${ }^{19}$. Les quelques concessions obtenues, comme la gestion collective des frontières, le partage des check points entre le PYD et le parti majoritaire local dans les zones " pacifiées » ont été ratifiées dans cet accord (illustration 5). Au cours de ce voyage, nous avons pu observer que des check points mixtes étaient présents entre Dêrik et Qamishlo. Cependant, en dépit de ces accords, les check points mixtes entre le PYD et le PDKS, pendant syrien du PDK irakien, n'ont jamais été mis en œuvre.

Illustration 5 - Face à la ville de Faysh Khabûr, au Kurdistan irakien, le premier check point kurde dans l'extrême Nord-Est du territoire syrien



Auteur: P. Torres, mai 2013.

Parmi les check points visés par ces accords, celui-ci (illustration 5) témoigne du déploiement du pendant syrien du PKK (le YPG) dans les premiers temps de la prise de contrôle du territoire par les forces kurdes. Les inscriptions à la gloire d'« Apo » Öcalan, la similitude des drapeaux avec ceux du PKK (les couleurs ont été inversées à la suite des accords d'Erbil) ou encore l'uniforme du martyr présenté sur l'affiche surplombant le check point sont autant d'éléments révélateurs des influences initiales de l'insurrection kurde en Syrie.

36 Ces dispositions ne sont pas dénuées d'intérêt pour le PYD qui y trouve, outre une assise locale, un moyen de masquer son appartenance au PKK aux yeux d'étrangers peu informés de la situation politique kurde. Ainsi, au sein du Conseil Suprême Kurde, s'affrontent les deux modèles de ce Kurdistan en devenir. Le PKK et le PDK irakien s'y 
livrent une guerre silencieuse, faite d'arrestations arbitraires, tortures et exécutions, sous le masque d'une bienveillantes collaboration.

« Nous travaillons ensemble, par-delà nos divergences, dans l'intérêt des Kurdes et du Rojava Kurdistan »

(entretien de l'auteur avec un journaliste de Ronahi TV, média affilié au PYD, rencontré dans une base des YPG de la ville de Girkê Legê, le 5 mai 2013).

Les accords d'Erbil ont été violés à plusieurs reprises par le PYD. L'arrestation dans la Jezireh par les forces du PYD de 67 militants d'El-Partî, un parti kurde syrien proche du PDK irakien, en mai $2013^{20}$, a haussé d'un cran les tensions entre le PYD et le PDK. Mais le PDK dispose de moyens de pression efficaces. Après avoir renforcé la présence militaire sur son sol, le Kurdistan irakien a fermé la frontière entre son territoire et la Syrie ${ }^{21}$ et obtenu la libération des militants arrêtés.

La discontinuité géographique des zones de peuplement kurdes, ainsi que l'existence de quartiers kurdes à Damas ou Alep, posent problème dans l'érection d'une région autonome. La question reste ouverte de savoir si l'hypothèse d'une homogénéisation des zones de peuplement kurde n'est pas à envisager. Les flux de réfugiés à destination du Kurdistan irakien, originaires de l'ensemble de la Syrie, ainsi que le repli communautaire observable en situation de guerre civile, pourraient contribuer à constituer une zone de peuplement kurde plus homogène et continue. Une telle mutation rendrait un contrôle de l'espace plus aisé pour les forces kurdes qui, pour le moment, ne bénéficient pas de l'appui turc. M. Barzani garde plus d'un atout; le spectre du nettoyage ethnique, conséquence envisageable de l'ambivalente stratégie kurde, ne peut qu'accroître le caractère précieux de son soutien aux yeux de la population kurde de Syrie. En dépit de son statut d'allié objectif, le Kurdistan d'Irak entretient également une rivalité avec le PYD lui permettant de peser dans la destinée du Kurdistan syrien. Cette rivalité se perçoit également dans le fait que le PDK forme militairement de centaines de jeunes kurdes de Syrie $^{22}$, alors que le PYD ne les laisse entrer en Syrie qu'à la condition qu'ils rejoignent leurs rangs. Des déclarations récentes de M. Barzani laissaient entendre la possibilité d'une intervention directe du KRG au Kurdistan syrien «dans le cas où la menace djihadiste se ferait trop pressante $»^{23}$.

Cette position permet au KRG de contrôler lui-même les voies de communications avec la Syrie. Conscient de son rôle déterminant dans l'évolution de la situation des Kurdes de Syrie, le gouvernement du KRG a bâti à Faysh Khabûr un pont temporaire (illustration 6) destiné au transit de biens humanitaires, de réfugiés et aux personnalités politiques à destination du Kurdistan syrien. En plus de ce pont, un réseau de câbles fournit un approvisionnement en électricité et un accès à Internet que le régime baathiste n'assure plus sans défaillance. 


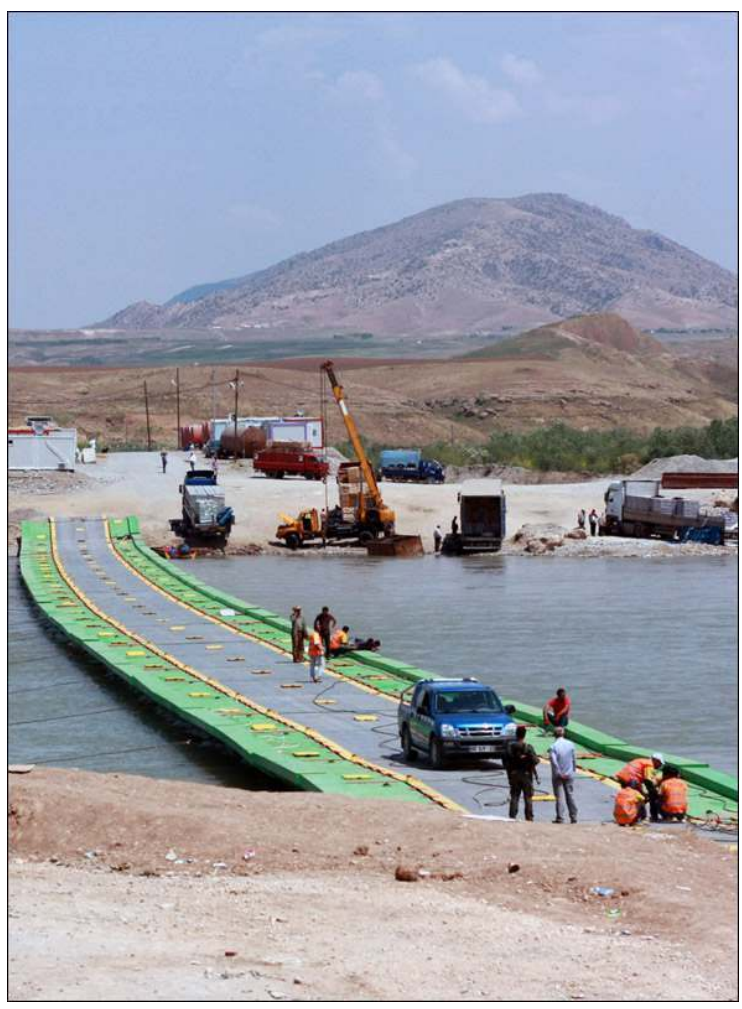

Auteur : P. Torres, mai 2013.

Ainsi, le Kurdistan occidental connait déjà une lutte d'influence entre ses deux modèles historiques, turc et irakien. Si l'on pouvait raisonnablement penser que les discussions tenues du 15 au 17 septembre 2013 entre les partis kurdes à Erbil ${ }^{24}$ pouvaient conduire à un règlement des tensions présentes, les rapports (entre janvier 2013 et octobre 2013, date de rédaction de cet article) sur les détentions d'opposants par le PYD montrent que l'accès au pouvoir dans la zone est d'ores et déjà un enjeu brûlant. La pression islamiste de plus en plus prononcée n'est pas plus urgente à traiter que la définition de rapports de forces en vue de l'accès au pouvoir une fois la paix revenue. Sur l'échiquier syrien, quelle que soit l'issue de la guerre entre l'armée loyaliste et les insurgés, les Kurdes de Syrie ont su prendre un ensemble de droits (notamment culturels) qui ne leur sont plus refusés.

Dans la perspective d'une fin des affrontements, on peut émettre trois hypothèses. Dans le cas où le régime de Damas stabiliserait la situation, on peut supposer que le pays ne serait pas en mesure de reconquérir les zones désormais contrôlées par les Kurdes. Un retour à la normale ne pourrait donc se faire qu'au prix de concessions qui entérineraient l'autonomie des Kurdes de la Jezireh. Si au contraire l'opposition venait à l'emporter, le soutien des Kurdes lui serait indispensable dans la lutte contre les éléments djihadistes qui s'opposent à la mise en place d'un État démocratique. Des concessions en faveur des Kurdes sont donc envisageables dans ce cadre précis. Si enfin la seule puissance en mesure de contrôler le territoire au terme de cette guerre était le front djihadiste, le Kurdistan d'Irak, soutenu par les États-Unis, ne manquerait pas d'intervenir pour protéger «ses frères kurdes", comme M. Barzani l'affirmait en août dernier ${ }^{25}$. Fait remarquable, on assiste dès à présent à une homogénéisation des zones de peuplement kurde, que le repli communautaire en temps de guerre civile ne peut qu'accélérer. 


\section{Conclusion}

La lutte pour le pouvoir au sein de la région autonome kurde sera, une fois la situation pacifiée, un excellent révélateur du poids respectif pris dans la guerre par les acteurs du changement à l'échelle régionale

Après deux années de guerre, l'aspiration à la liberté fait progressivement place parmi les populations syriennes à un désir de sécurité. Les unités de Défense du Peuple, quel que soit leur degré de légitimité, semblent en mesure de fournir cette sécurité dans la majeure partie des zones kurdes de Syrie via l'imposition de leur vision du Kurdistan à venir, sensiblement modulée par les jeux diplomatiques des Kurdes d'Irak. Au vu de ces influences kurdes et cependant étrangères, il semble que la situation tende à bâtir un Kurdistan de frontières, formé de régions autonomes intégrées les unes aux autres au sein d'États ayant revu à la baisse leurs prétentions hégémoniques, par une relative décentralisation des formes de contrôle et d'exploitation des ressources. La viabilité de ce Kurdistan " en creux » reposerait sur des relations transfrontalières intensifiées. Cette tendance à un dépassement, au moins temporaire, du modèle de l'État-nation se retrouve dans les discours de bien des forces politiques kurdes. Par exemple, Saleh Muslim, leader du PYD déclarait : « Il y a un printemps kurde. Une organisation kurde transnationale doit voir le jour mais le modèle de l'État-nation est dépassé. »(Le Monde, 27 août 2013). Ce scénario et les voies pour y parvenir semblent pouvoir répondre à des nécessités contradictoires, soit en permettant au régime de Damas de rasseoir sa clientèle à moindre frais, soit en fournissant à l'ASL un allié de poids, tant du point de vue militaire que politique et informationnel, à la seule condition d'intégrer le désir d'autodétermination des Kurdes au sein de la Syrie de demain.

\section{BIBLIOGRAPHIE}

Barzani S., 2008. Géopolitique actuelle des Kurdes en Turquie, en Irak et en Iran. EchoGéo [en ligne], Sur le vif, http://echogeo.revues.org/2380

Boulanger P., 2006. Géopolitique des Kurdes. Paris, Ellipses.

Bozarslan H., 1997. La question kurde : États et minorités au Moyen-Orient. Paris, Presses de Sciences Po.

Burgat F., 2013. Le printemps syrien au prisme de ses prédécesseurs. A paraître in Un printemps arabe ? Presses de l'Université de Rennes, hal/shs, mis en ligne le 29 novembre 2012, http:// halshs.archivesouvertes.fr/docs/00/75/86/08/PDF, page consultée le 8 septembre 2013.

Galula D., 2008. Contre-insurrection : théorie et pratique. Paris, Économica.

Gauthier J., 2006. Syrie : le facteur kurde. Outre-Terre, vol. 14, nº 1, p. 217-231.

Park B., 2012. Turkey, the US and the KRG: Moving Parts and the Geopolitical Realities. Insight Turkey, vol. 14, $\mathrm{n}^{\circ} 3$ [en ligne]. 
Scalbert-Yücel C., Tejel J. (dir.), 2010. Ruralité, urbanité et violence au Kurdistan. Études rurales, n ○ 186, numéro thématique, Paris, EHESS.

Tejel Gorgas J., 2006. Les Kurdes de Syrie, de la "dissimulation" à la "visibilité" ? Revue des mondes musulmans et de la Méditerranée [en ligne], n 115-116, http://remmm.revues.org/3022

Tejel Gorgas, J., 2009. Syria's Kurds: History, Politics and Society. Londres, Routledge.

Yazbek S., 2012. Feux croisés : journal de la révolution syrienne. Paris, Buchet Chastel.

\section{NOTES}

1. Irénées.net, 2006. L'élaboration et l'utilisation de l'identité dans la construction d'un conflit : la question Kurde en Turquie. En ligne, page consultée le 8 septembre 2013, http:// www.irenees.net/bdf_fiche-analyse-644_fr.html

2. Westal S., Jambaz M., 2013. Kurdish refugees trek to northern Iraq over parched hills. En ligne, page consultée le 13 septembre 2013, http://www.dailystar.com.lb/News/Middle-East/2013/ Aug-27/228793-kurdish-refugees-trek-to-northern-iraq-over-parched-hills.ashx.

3. Arango T., 2013. Syrian Kurds Find More Than a Refuge in Northern Iraq. NY Times, 23 Août 2013, en ligne, page consultée le 13 septembre 2013, http://www.nytimes.com/2013/08/24/ world/middleeast/syrian-kurds-find-more-than-a-refuge-in-iraqi-kurdistan.html? pagewanted=all\&_r=0

4. Institut Kurde de Paris, 2009. Erdogan promet des réformes pour les Kurdes d'ici la fin de l'année. En ligne, page consultée le 8 Septembre 2013, http://www.institutkurde.org/info/depeches/ erdogan-promet-des-reformes-pour-les-kurdes-d-ici-la-fin-de-l-annee-2127.html

5. Erlich R., 2011. In Syria, Kurds Split Over Support for Assad Regime. Pulitzer Center, http:// pulitzercenter.org/reporting/syria-kurdish-groups-armed-forces-assad-controversy

6. AFP, 2011. Syrie: assassinat du dirigeant et opposant kurde Mechaal Tamo. Le Point, en ligne, page consultée le 8 septembre 2013, http://www.lepoint.fr/monde/syrie-assassinat-dudirigeant-et-opposant-kurde-mechaal-tamo-07-10-2011-1382053_24.php

7. http://www.kurdishglobe.net/article/B401BD71512EFC6525D69EA3048CD6F7/Syria-s-Kurdsare-they-about-to-join-the-uprising-against-Assad-.html

8. La représentante du PYD à Bruxelles écarte ces accusations comme une intoxication turque : « la Turquie a excité les populations arabes syriennes en leur faisant croire que le PYD avait signé des accords avec le régime, en faisant des traîtres à la révolution. Le départ précipité des forces militaires loyalistes de la plupart des zones kurdes n'était lié qu'à la nécessité stratégique de les concentrer sur des fronts jugés plus urgents (...) », in Mellek Rodhi, 2013. Il n'y aura aucune solution à la crise syrienne sans résolution du dossier kurde. Jeune Afrique, en ligne, page consultée le $\quad 9$ septembre 2013, http://www.jeuneafrique.com/Article/ ARTJAWEB20130614151817/

9. France 24, 6 septembre 2012. Les Kurdes syriens donnent des sueurs froides à Ankara. En ligne, page consultée le 11 septembre 2013, http://www.france24.com/fr/20120803-syrie-turquiekurdes-pkk-nord-assad-crise-frontieres-armee-erdogan-kurdistan

10. RFI, 29 Juin 2013. Syrie : couvre-feu imposé à Amouda dans le Kurdistan syrien. En ligne, page consultée le 9 septembre 2013, http://www.rfi.fr/moyen-orient/20130629-syrie-couvre-feuimpose-amouda-le-kurdistan-syrien ; Kurdwatch, 27 juin 2013.'Amudah: Hunger strike against PYD-attacks. En ligne, page consultée le 9 septembre 2013 http://kurdwatch.org/index.php? aid $=2888 \& z=e n \&$ cure $=1009$ 
11. L'Express, 7 avril 2011. Damas naturalise 300000 kurdes. En ligne, page consultée le 9 septembre 2013 , http://www.lexpress.fr/actualite/monde/la-syrie-naturalise-300-000kurdes_980532.html

12. Kurdwatch, mai 2013. Damascus: Kurdish Literature as a field of study. En ligne, page consultée le 11 septembre 2013, http://www.kurdwatch.org/index.php? aid $=2819 \& z=$ en $\&$ cure $=1009$

13. Kurdwatch, 9 décembre 2012. Amudah: PYD destroys Military Intelligence Service documents. En ligne, page consultée le 12 Septembre 2013, http://www.kurdwatch.org/? aid $=2706 \& \mathrm{z}=$ en\&cure $=245$

14. Cantener A., 8 mai 2013. Retrait des rebelles du PKK : la réconciliation turco-kurde va être la question à venir. Rfi.fr, en ligne, page consultée le 11 septembre 2013, http://www.rfi.fr/ europe/20130508-retrait-rebelles-pkk-reconciliation-turco-kurde-syrie-iran-irak; Le Nouvel Observateur, 11 septembre 2013. Turquie : le PKK appelle à boycotter les écoles, soutenir les manifestations. En ligne, page consultée le 11 septembre 2013, http://tempsreel.nouvelobs.com/ education/20130911.AFP4964/turquie-le-pkk-appelle-a-boycotter-les-ecoles-soutenir-lesmanifestations.html

15. Williams L., 15 août 2013. Islamist militants drive Free Syrian Army out of Raqqa. The Daily Star, en ligne, page consultée le 11 septembre 2013, http://www.dailystar.com.lb/News/MiddleEast/2013/Aug-15/227444-islamist-militants-drive-free-syrian-army-out-of-raqqa.ashx 16. Weiss M., 5 novembre 2012. The impending Syrian-Kurdish conflict. The Henry Jackson Society, en ligne, page consultée le 11 septembre 2013, http://henryjacksonsociety.org/2012/11/05/theimpending-syrian-kurdish-conflict/

17. Mardini B., 27 août 2013.Les Kurdes se rapprochent du reste de l'opposition. Elaph, en ligne, page consultée le 11 septembre 2013, http://www.courrierinternational.com/ article/2013/08/27/les-kurdes-se-rapprochent-du-reste-de-l-opposition

18. Haski P., 2 août 2013. Kurdes vs djihadistes, la guerre dans la guerre en Syrie. Rue89, en ligne, page consultée le 11 septembre 2013, http://www.rue89.com/2013/08/02/kurdes-vs-djihadistesguerre-guerre-syrie-244716 ; Stuart H., 31 juillet 2013. Kurdish militants vs. al-Qaeda - Syria's war within a war. The Henry Jackson Society, en ligne, page consultée le 11 septembre 2013, http:// henryjacksonsociety.org/2013/07/31/kurdish-militants-vs-al-qaeda-syrias-war-within-a-war/

19. Tanir I., Van Wilgenburg W., Hossino O., 2012. Unity or PYD power play? Syrian kurdish dynamics after the Erbil Agreement. The Henry Jackson Society, en ligne, page consultée le 12 septembre 2013, http://henryjacksonsociety.org/wp-content/uploads/2012/10/HJS_Unity-orPYD-Power-Play_-Report.pdf

20. Kurdwatch, 21 mai 2013. Al-Qamishli: YPG kidnaps sixty-seven members of el-Partî. En ligne, page consultée le 12 novembre 2013, http://www.kurdwatch.org/index.php? aid $=2837 \& z=$ en \&cure $=1009$

21. Kurdwatch, 26 mai 2013. Faysh Khabur: Conflict between PYD and KRG escalates. En ligne, page consultée le 12 novembre 2013, http://www.kurdwatch.org/index.php? aid $=2820 \& z=e n \&$ cure $=1009$

22. Tanir I., Van Wilgenburg W., Hossino O., 2012. Unity or PYD power play? Syrian kurdish dynamics after the Erbil Agreement. The Henry Jackson Society, en ligne, page consultée le 12 septembre 2013, http://henryjacksonsociety.org/wp-content/uploads/2012/10/HJS_Unity-orPYD-Power-Play_-Report.pdf; Today's Zaman, 2013. Syrian Kurds given military training in northern Iraq, says Barzani. World Security Network, en ligne, http:// www.worldsecuritynetwork.com/Syria/Todays-Zaman/Syrian-Kurds-given-military-training-innorthern-Iraq-says-Barzani

23. Al Jazeera, 10 août 2013. Iraqi Kurd leader vows to defend Syria Kurds. En ligne, page consultée le 12 septembre 2013, http://www.aljazeera.com/news/ middleeast/2013/08/2013810192811431562.html 
24. Blog Le Monde, Au fil du Bosphore, 27 août 2013. Trois regards sur la situation au Kurdistan syrien. En ligne, page consultée le 12 septembre 2013, http:// istanbul.blog.lemonde.fr/2013/08/27/trois-regards-sur-la-situation-au-kurdistan-syrien/

25. Shekhani, S., 2 août 2013. En ligne, page consultée le 11 septembre 2013, http:// www.aawsat.net/2013/08/article55313186

\section{AUTEUR}

\section{FÉLIX POYER}

Félix Poyer, felix.poyer@univ-paris8.fr, est géographe et photographe. Il travaille actuellement comme cartothécaire à l'Université Paris 8. 